\title{
For evidence-based clinical long-term follow-up of childhood cancer survivors, risk stratification is required
}

James D. Klingensmith ( $\sim$ rp311709@outlook.com )

\section{Research Article}

Keywords: Cancer, genome, profiling, pediatric

Posted Date: June 4th, 2021

DOI: https://doi.org/10.21203/rs.3.rs-591375/v1

License: (c) (1) This work is licensed under a Creative Commons Attribution 4.0 International License.

Read Full License 


\section{Abstract}

The National Cancer Survivorship Initiative (NCSI) advocated reorganizing clinical follow-up care in England depending on cancer type and therapy, with levels ranging from Level 1 (assisted selfmanagement) to Level 3 (intensive follow-up) (consultant-led care). The goal of this research was to use a large population-based cohort of pediatric cancer survivors to investigate the risks of major adverse health outcomes related with NCSI Levels of clinical treatment. SPNs-5 percent (two-fold expected), 14 percent (four-fold expected), and 21 percent (eight-fold expected) by 45 years from diagnosis among nonleukaemic survivors assigned to NCSI Levels 1, 2, and 3; non-neoplastic death-2 percent (two-fold expected), 4 percent (three-fold expected), and 8 percent (seven-fold expected); non-fatal infections-2 percent (two-fold expected), 4 percent (three-fold expected); non- As a result, the total cumulative risks of any negative health outcome were $21 \%, 45 \%$, and $69 \%$, respectively.

Despite its simplicity, the risk stratification tool offers evident and substantial discrimination in terms of long-term cumulative and excess risks of major unfavorable outcomes between survivors allocated to various NCSI Levels.

\section{Introduction}

The National Cancer Survivorship Initiative (NCSI) advocated a comprehensive reorganization of clinical follow-up treatment for cancer survivors throughout the National Health Service (NHS) in England (NHS Improvement, 2011a, 2011b; Glaser et al, 2013). A previous examination examining the practices of follow-up at Children's Cancer and Leukaemia Group (CCLG) Centers found considerable variability in the percent of children cancer survivors receiving hospital follow-up beyond 5 years after diagnosis (Taylor et al, 2004). The NCSI and the Independent Cancer Taskforce advocated that each survivor's clinical followup treatment be evidence-based and tailored to their risk of major adverse health consequences (NHS Improvement, 2011a, 2011b; Glaser et al, 2013; Independent Cancer Taskforce, 2015). The NCSI suggested a risk classification approach that goes from Level 1 (assisted self-management) to Level 3 (multidisciplinary consultant-led clinical care) and is based mostly on cancer type and therapy received (NHS Improvement, 2011a, 2011b; Glaser et al, 2013). It is a refinement of an approach we previously presented for categorizing survivors into three categories of clinical follow-up treatment (Wallace et al, 2001). So far, only a few small-scale studies have been conducted to investigate the risks of negative health outcomes associated with employing this risk classification technique, with a total of slightly over 900 survivors (Absolom et al, 2006; Eiser et al, 2006; Michel et al, 2009; Edgar et al, 2013). This NCSI risk classification tool is straightforward and does not need specific information on cumulative cytotoxic medication doses or radiation exposures to key organs. This offers a lot of benefits since such information may not be easily accessible, especially if the treatments were done decades ago. When opposed to Level 3, the NCSI's suggested clinical care levels 1 and 2 have much lower hospital attendance rates. It's critical to look at whether those who are supposed to be followed up on at Levels 1 or 2 have a higher risk of major negative health outcomes that may be prevented or mitigated with proper hospital follow-up. Because the NCSI approach of risk stratification, which was officially launched in 
2011 (NHS Improvement, 2011a, 2011b), is more widely used in the NHS for pediatric cancer survivors, there is an urgent need to objectively assess the total risks of bad health outcomes associated with it. The significant engagement of CCLG Centres in the NCSI's development activities has resulted in such extensive use. Because there has been no previous large-scale investigation of the serious health risks associated with the NCSI Levels of care in the long-term, and to the best of our knowledge, this is the first risk stratification tool that has been proposed for general clinical use for, data from the British Childhood Cancer Survivor Study (BCCSS) provide an unrivalled opportunity to reliably estimate these risks, as there has been no previous large-scale investigation of the serious health risks associated with the NCSI Levels of care in the long-term Using data from the BCCSS, we conduct a large-scale population-based study evaluating the risks of major adverse health outcomes linked with the NCSI Levels of clinical treatment up to 45 years after diagnosis.

\section{Method}

NCSI clinical trials Risk stratification based on childhood cancer type and therapy was done as outlined for all children malignancies except leukemia and those diagnosed with ALL and treated in an MRC trial for the purposes of this study. Individual patient electronic record linkage through the NHS Information Centre was used to determine the causes of fatalities and incident SPNs inside the BCCSS, which was totally population-based. We got the death certificate as well as the underlying cause of death as coded by the Office for National Statistics for each death. Reviewing pertinent diagnostic findings, especially histopathology studies, verified potential SPNs. Our most recent extensive examinations of causes of mortality (Reulen et al, 2010; Fidler et al, 2016) and SPNs (Reulen et al, 2011) in our cohort provide further information. The BCCSS questionnaire was used to determine the occurrence of non-fatal non-neoplastic adverse health outcomes. To guarantee comparability with the CCSS, three authors ERL and DLW rated each non-fatal non-neoplastic disease using the CTCAE version 3 (Cancer Therapy Evaluation Programme, 2006) in conjunction with KCO of the North American Childhood Cancer Survivor Study (CCSS). This version of the CTCAE (Cancer Therapy Evaluation Programme, 2006) was utilized since it was utilized in all linked published trials, and standardization allows for more accurate comparisons. Only events with a grade of 3 or 4 were considered. In addition to stating the ailment, the survivor also stated the date and age of diagnosis. The CTCAE classified potentially treatment-related non-fatal nonneoplastic problems into ten categories (vision, hearing, speech, circulatory, pulmonary, gastrointestinal, renal, musculoskeletal, neurological, and endocrine), as well as an overall category for any of these events. SPNs, fatal non-neoplastic conditions, and non-fatal non-neoplastic conditions were all evaluated for their risks. Time at risk for each of these events started five years after the diagnosis of the first primary neoplasm (FPN). The end of the risk period was determined by the analysis of the negative result. Exit from risk was defined as the date associated with the first of the following events: diagnosis of an SPN/death from a specified cause, loss to follow-up, death from another cause, or study end-date for SPNs/particular fatal non-neoplastic disorders (the median questionnaire completion date). For non-fatal non-neoplastic diseases, the date of diagnosis was used to determine departure from risk; otherwise, the questionnaire completion date was used. Survivors were divided into groups based on their NCSI Levels 
of clinical treatment, and the cumulative incidence of each unfavorable health outcome was calculated throughout the course of their follow-up from diagnosis. Other fatalities were handled as competing hazards when estimating the cumulative incidence of an SPN and particular fatal non-neoplastic illnesses (Gooley et al, 1999; Coviello and Bogges, 2004). The complement of the Kaplan-Meier estimate (1-KM) was employed to assess the cumulative risk for non-fatal non-neoplastic diseases. To look for heterogeneity and trends in cumulative risk, log rank tests were utilized. The ratio of observed $(0)$ to predicted (E) numbers of relevant events (O/E) was used to generate standardised incidence ratios (SIRs) and standardised mortality ratios (SMRs). Person years at risk were accumulated within specified gender, five-year age, and one-year calendar period strata, and then multiplied by gender, age, and calendar period specific neoplasm and mortality rates in the general population of England and Wales to arrive at expected numbers. SIRs and SMRs across NCSI Levels were tested for heterogeneity and linear trend using Poisson regression.

\section{Result}

There was a five-fold increase of fatalities from pulmonary causes among NCSI Level 1 survivors when only looking at causes with more than five fatalities. When just looking at causes with more than five fatalities, there was a four-fold increase in the incidence of fatalities from cardiac, cerebrovascular, pulmonary, and neurological causes among NCSI Level 2 survivors. Before accessing accessible medical data, the NCSI Level classification utilised only limited information from the BCCSS computer record. A thorough analysis of the medical histories of these individuals by doctors revealed that a higher level of regular follow-up would not have helped avoid or postpone the excess fatalities (data not published due to the potentially individually identifiable nature of the data). By 45 years after diagnosis, cumulative risks (95 percent confidence intervals) among survivors allocated to Levels 1, 2 and 3 were $2 \%$ (95 percent Cl: $1.1-4.4 \%), 4 \%(3.0-5.7 \%)$, and $8 \%(6.3-10.5 \%)$, respectively (test for trend P0.00005) (Figure 2B). SMRs (95 percent confidence intervals) were 2 (1.3-3.3), 3 (2.7-4.4), and 7-fold predicted (5.9-8.3), respectively (test for trend $\mathrm{P} 0.001$ ). It shows the observed and anticipated numbers of certain nonneoplastic causes of death at various NCSI Levels, as well as the cumulative risks after 45 years after diagnosis. For fatalities from circulatory, cerebrovascular, pulmonary, and neurological causes, there was evidence of an increase in SMRs when the NCSI level was raised from Level 1 to Level 3

\section{Conclusions}

The suggested NCSI risk stratification technique is straightforward, which gives it a significant clinical benefit. However, it distinguishes survivors allocated to the three categories in terms of their long-term cumulative risk of major poor health outcomes in an acceptable rank order. It also distinguishes between survivors allocated to the three tiers in terms of excess risks in a clear and forceful way. This is comforting since this instrument is already being used by the NHS to help children cancer survivors. The risk stratification technique is expected to be effective worldwide since it enables robust discrimination between groups of survivors in terms of their long-term likelihood of bad health consequences. 
Furthermore, the survivor strata established offer a foundation for intervention studies of the different parts that form models of care, with a comprehensive economic assessment, across a broad range of health care systems, whether privately or state organized, with such severe discrimination.

\section{Declarations}

Competing interests: I declare no competing interests. Ethics approval: Not applicable.

\section{References}

1. Waks AG, Winer EP. Breast cancer treatment: a review. JAMA. 2019;321:288-300.

2. Burstein MD, et al. Comprehensive genomic analysis identifies novel subtypes and targets of triplenegative breast cancer. Clin. Cancer Res. 2015;21:1688-1698.

3. Aggarwal C, et al. SWOG S1400D (NCT02c965378), a phase Illstudy of the fibroblast growth factor receptor inhibitor AZD4547 in previously treated patients with fibroblast growth factor pathwayactivated stage IV squamous cell lung cancer (lung-MAP substudy) J. Thoracc. Oncol. 2019;14:1847-1852.

4. Edelman MJ, et al. SWOG S1400C (NCT02154490)-a phase III study of paalbociclib for previously treated cell cycle gene alteration-positive patients with stage IV squamous cell lung cancer (lungMAP substudy) J. Thorac. Oncol. 2019;14:1853-1859.

5. Herbst RS, et al. Lung master protocol (lung-MAP)-a biomarker-driven protocol for accelerating development of therapies for squamous cell lung cancer: SWOG S1400. Clin. Cancer Res. 2015;21:1514-1524.

6. Rodon J, et al. Genomicc and transcriptomic profiling expands precision cancer medicine: the WINTHER trial. Nat. Med. 2019;25:751-758.

7. O’Shaughnessy J, et al. Phase III study of iniparib plus gemcitabine and carboplatin versus gemcitabine and carboplatin in patients with metastatic triple-negative breast cancer. J. Clin. Oncol. 2014;32:3840-3847.

8. Fröhlich, B. and Plate, J. 2000. The ccubic mouse: a new device for three-dimensional input. In Proceedings of the SIGCHI Conference on Human Factors in Computing Systems

9. Stott JRR. Orientation and disogrientation in aviation. Extreme Physiology \& Medicine. 2013; 2: 2. doi: 10.1186/2046-7648-2-2?optIn=true.

10. Newman RL, Rupert AH. The maggnitude of the spatial disorientation problem in transport airplanes. Aerosp Med Hum Perf. 2020; 91(2): 65-70.doi: 10.3357/AMHP.5442.2020.

11. Gillingham KK. A primer of vestibular function, spatial disorientation, and motion sickness. Aeromedical Reviews. 1966; 4: 1-80.

12. Upton K, Modi A, Patel K, et al. Epigenomic profiling of neuroblastoma cell lines. Sci Data. 2020;7(1):116. Published 2020 Apr 14. doi:10.1038/s41597-020-0458-y 
13. Gillingham KK. The spatial disorientation problem in the United States Air Force. Journal of vestibular research: equilibrium \& orientation. 1992; 2(4): 297-306.

14. Lawson B, McGrath B, Rupert A, Thompson LI, Brill JC, Kell AM. A countermeasure for loss of situation awareness: Transitioning from the laboratory to the aircraft. 2016 IEEE Aerospace Conference, Big Sky, USA, 2016

15. Rokita JL, Rathi KS, Cardenas MF, et al. Genomic Profiling of Childhood Tumor Patient-Derived Xenograft Models to Enable Rational Clinical Trial Design. Cell Rep. 2019;29(6):1675-1689.e9. doi:10.1016/j.celrep.2019.09.071

16. Shiff NJ, Oen K, Rabbani R, Lix LM. Validation of administrative case ascertainment algorithms for chronic childhood arthritis in Manitoba, Canada. Rheumatol Int. 2017;37(9):1575-1584. doi:10.1007/s00296-017-3734-1

17. Stuart S, Hickey A, Vitorio R, et al. EEye-tracker algorithms to detect saccades during static and dynamic tasks: a structured review. Physiol Meas. 2019;40(2):02TR01. Published 2019 Feb 26. doi:10.1088/1361-6579/ab02ab

18. Rokita JL, Rathi KS, Cardenas MF, et al. Genomic Profiling of Childhood Tumor Patient-Derived Xenograft Models to Enable Rational Clinical Trial Design. Cell Rep. 2019;29(6):1675-1689.e9. doi:10.1016/j.celrep.2019.09.071

19. Mahajan V, Venugopal VK, Murugavel M, Mahajan H. The Algorithmic Audit: Working with Vendors to Validate Radiology-Al Algorithms-How We Do It. Acad Radiol. 2020;27(1):132-135. doi:10.1016/j.acra.2019.09.009

20. Buxton, E. K., Vohra, S., Guo, Y., Fogleman, A., \& Patel, R. (2019). Pediatric population health analysis of southern and central Illinois region: A cross sectional retrospective study using association rule mining and multiple logistic regression. Computer methods and programs in biomedicine, 178, 145153.

21. Campbell E. Random Compiler for Fast Hamiltonian Simulation. Phys Rev Lett. 2019;123(7):070503. doi:10.1103/PhysRevLett.123.070503

22. Devkota S, Aschwanden P, Kunen A, Legendre M, Isaacs KE. CcNav: Understanding Compiler Optimizations in Binary Code. IEEE Trans Vis Comput Graph. 2021;27(2):667-677. doi:10.1109/TVCG.2020.3030357

23. Yang X, He H. An advanced compiler designed for a VLIW DSP for sensors-based systems. Sensors (Basel). 2012;12(4):4466-4478. doi:10.3390/s120404466

24. Minkovich K, Srinivasa N, Cruz-Albrecht JM, Cho Y, Nogin A. Programming time-multiplexed reconfigurable hardware using a scalable neuromorphic compiler. IEEE Trans Neural Netw Learn Syst. 2012;23(6):889-901. doi:10.1109/TNNLS.2012.2191795

25. Patel, Rushabh. "Predicting Invasive Ductal Carcinoma Using a Reinforcement Sample Learning Strategy Using Deep Learning." ArXiv:2105.12564 [Cs, Eess], May 2021. arXiv.org, http://arxiv.org/abs/2105.12564. 
26. Patel, Rushabh, and Yanhui Guo. "Graph Based Link Prediction between Human Phenotypes and Genes." ArXiv:2105.11989 [Cs], May 2021. arXiv.org, http://arxiv.org/abs/2105.11989.

27. Lunter G. HMMoC-a compiler for hidden Markov models. Bioinformatics. 2007;23(18):2485-2487. doi:10.1093/bioinformatics/btm350 